\title{
Berkeley Off-line Radioisotope Generator (BORG)
}

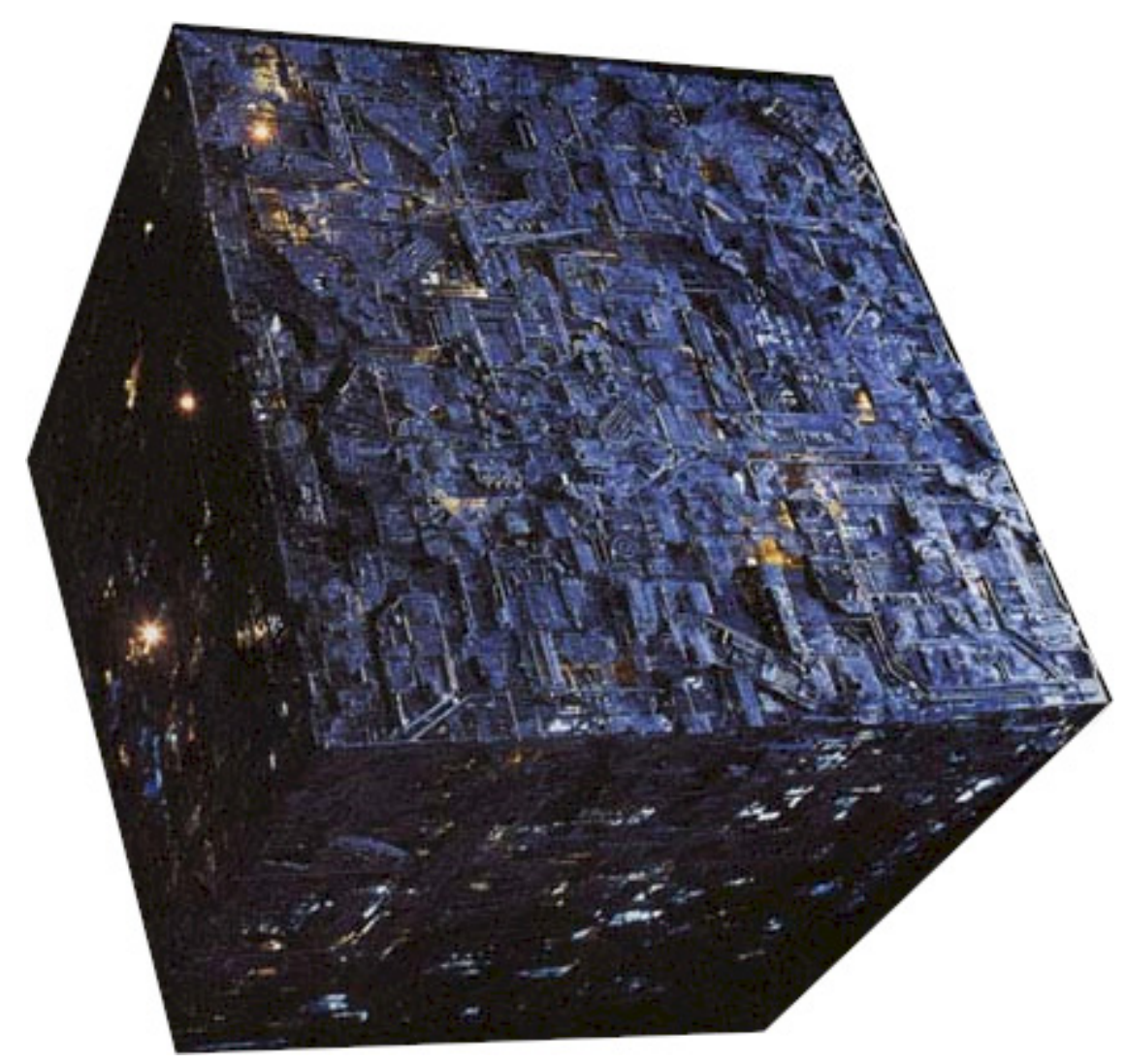

Ralf Sudowe

Joshua Patin

July 23, 2001 


\section{Introduction}

Development of chemical separations for the transactinides has traditionally been performed with longer-lived tracer activities purchased commercially. With these longlived tracers, there is always the potential problem that the tracer atoms are not always in the same chemical form as the short-lived atoms produced in on-line experiments. This problem is especially severe for elements in groups 4 and 5 of the periodic table, where hydrolysis is present. The long-lived tracers usually are stored with a complexing agent to prevent sorption or precipitation. Chemistry experiments performed with these longlived tracers are therefore not analogous to those chemical experiments performed in online experiments. One way to eliminate the differences between off-line and on-line chemistry experiments is through the use of a ${ }^{252} \mathrm{Cf}$ fission fragment collection device.

A ${ }^{252} \mathrm{Cf}$ fission fragment collection device has already been constructed [1]. This device is limited in its capabilities. A new fission fragment device would allow the study of the chemical properties of the homologues of the heaviest elements. This new device would be capable of producing fission fragments for fast gas chemistry and aqueous chemistry experiments, long-lived tracers for model system development and neutrons for neutron activation. Fission fragment activities produced in this way should have the same chemical form as those produced in Cyclotron irradiations. The simple operation of this source will allow more rapid and reliable development of radiochemical separations with homologues of transactinide elements.

\section{Chemistry}

\section{A. Significance}

The production of specific, carrier free radionuclides with short and medium halflives plays an important role in studying the transactinide elements. Over the last 15 years large effort was devoted to this research area, the elements $\mathrm{Rf}$ and $\mathrm{Db}$ have been studied extensively, and the studies were extended to $\mathrm{Sg}, \mathrm{Bh}$ and recently to $\mathrm{Hs}$ as well.

To study the chemical behavior of the transactinide elements it is necessary to do experiments with the chemical homologues first. Using the homologues, an appropriate chemical system for on-line experiments can be chosen. In addition by studying the homologues, a base set of data can be obtained to which the chemical behavior of the transactinides can be compared afterwards.

The radioactive isotopes of the homologue elements can either be bought commercially, produced by neutron irradiation in a reactor or in nuclear reactions at an accelerator. Another method to obtain the nuclides is by using a source containing a radionuclide that undergoes spontaneous fission. In the latter case the fission products can be separated from the source and used as tracers.

If the radionuclides are bought commercially, they are usually only available in solution. In this case hydrolysis of the tracer can be a problem, and if other ions like fluoride are added to the solution to prevent hydrolysis, they might interfere with the planned experiments. In addition most of the radionuclides are very expensive when bought and only radionuclides with longer half-lives are available. 
The reaction products of neutron irradiations are normally not carrier free, due to the large amounts of material that is irradiated. They need to be separated very thoroughly from the matrix material, a process that usually is very complicated and time consuming. Short-lived radiotracers can't be obtained by this method; the available range of half-lives is given by the time necessary for the separation.

The production of radionuclides in reactions involving targets and heavy ions beams is very useful in obtaining short-lived carrier free radioisotopes. The lifetime of the available nuclides is only limited by the transport time from the target to the chemistry site, and since the reaction products recoil from the target and are transported using a gas jet system, they are carrier free. Unfortunately these experiments need to make use of large accelerators or cyclotrons, which means the tracers can only be produced when beam time is available.

\section{B. History}

\section{Gas phase chemistry}

Carrier free radionuclides play an important role in studying the chemical behavior of the transactinide elements in the gas phase. The gas phase chemistry of Rf, $\mathrm{Db}, \mathrm{Sg}, \mathrm{Bh}$ and Hs has been studied using different techniques [2-5].

To optimize the experimental conditions for the experiments and to test the efficiency of the chemical separation work with the homologue elements is necessary. None of the commercially available nuclides can be used as tracers, because they are usually shipped in solutions and an efficient transfer of these nuclides into the gas phase is extremely difficult. Gas phase chemistry allows for studying transactinide elements with short halflives on the order of a few seconds. This means that the homologues of interest should be studied using isotopes with similar half-lives. This limits the production of the radiotracers to either nuclear reactions involving accelerators and the subsequent transport of the reaction products using a gas jet, or using a fission source.

For example Tc and Rh, the homologues for element 107, Bh have been studied using the Tc isotopes ${ }^{101} \mathrm{Tc}$ and ${ }^{104} \mathrm{Tc}$ and the $\mathrm{Rh}$ isotopes ${ }^{183} \mathrm{Rh}$ and ${ }^{184} \mathrm{Rh}$. The $\mathrm{Rh}$ isotopes were obtained by bombarding a ${ }^{\text {nat }} \mathrm{W}$ target with protons, while the $\mathrm{Tc}$ isotopes were obtained as fission products from a ${ }^{252} \mathrm{Cf}$ source at the PSI in Switzerland [6, 7].

\section{Aqueous phase chemistry}

Model systems for the study of transactinide elements in solution were generally developed using radiotracers with medium half-lives. Typical radiotracers like ${ }^{95} \mathrm{Zr},{ }^{95} \mathrm{Nb}$ or ${ }^{131} \mathrm{Sn}$ were either bought commercially or produced by irradiating samples in the neutron flux of a reactor and separating the activation products afterwards [8]. Other radiotracers like ${ }^{169} \mathrm{Hf}$ could only be produced by irradiating targets with heavy ion beams. Afterwards the reaction products needed to be transported from the target using a gas jet system [9].

One automated chemistry apparatus, SISAK, is designed to work with nuclides with half-lives of a few seconds [10]. To optimize these systems and to study the behavior of the homologues under the same conditions, the radionuclides used in test experiments 
should have half-lives in the same range. These nuclides could usually only be produced in heavy ion reactions. These experiments were therefore limited to the beam time available at the accelerator [11].

\section{Goals}

The proposed radionuclide generator should be capable of delivering radionuclides that can be used to develop model systems to study the chemical behavior of transactinide elements. The ${ }^{252} \mathrm{Cf}$ source should produce nuclides with half-lives on the order of a few seconds as well as nuclides with half-lives on the order of hours or weeks that can be used as tracers in experiments.

\section{Overview}

\section{1. ${ }^{252} \mathrm{Cf}$ as a source of fission products}

Based on experiences with previous generations of fission product generators, ${ }^{252} \mathrm{Cf}$ has been proven to be good choice as source material $[1] .{ }^{252} \mathrm{Cf}$ has a half-life of 2.6 years. It decays by alpha decay $97 \%$ of the time and by spontaneous fission $3 \%$ of the time. The short half-life of the nuclide limits the lifetime of a ${ }^{252} \mathrm{Cf}$ source to about 5 to 8 years. While this means that the source inside the generator needs to be replaced in regular intervals, it also means that the source has a reasonable high rate of fission events. Other nuclides that undergo spontaneous fission with a longer half-life do not provide the same amount of fissions as a ${ }^{252} \mathrm{Cf}$ source and are not as easily available. Therefore substantially more material would be needed when plating the source.

As can be seen from Fig. 1, the light branch of the fission product distribution includes nuclides with mass numbers ranging from 90 to about 120 . The heavy branch includes nuclides with mass numbers ranging from 130 to 160 . 


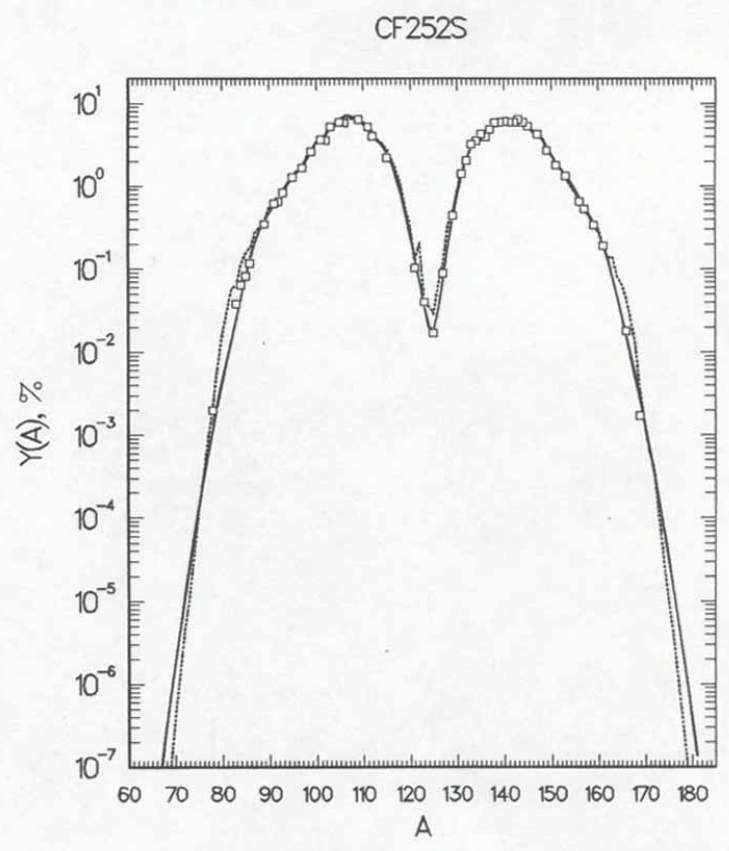

Fig. 1. Mass-number yields $\mathrm{Y}(\mathrm{A})$ vs. mass number A. $\square$ : experimental data for ${ }^{252} \mathrm{Cf}$; - : calculated from the $A_{p}$ model; ---: Rider's mass number yields [12].

Fig. 2 shows that the spontaneous fission of ${ }^{252} \mathrm{Cf}$ yields mainly short-lived isotopes of the elements with proton numbers ranging from 35 to 45 and from 55 to 65 . These primary fission products then undergo beta decay to yield longer-lived radionuclides and finally stable isotopes.

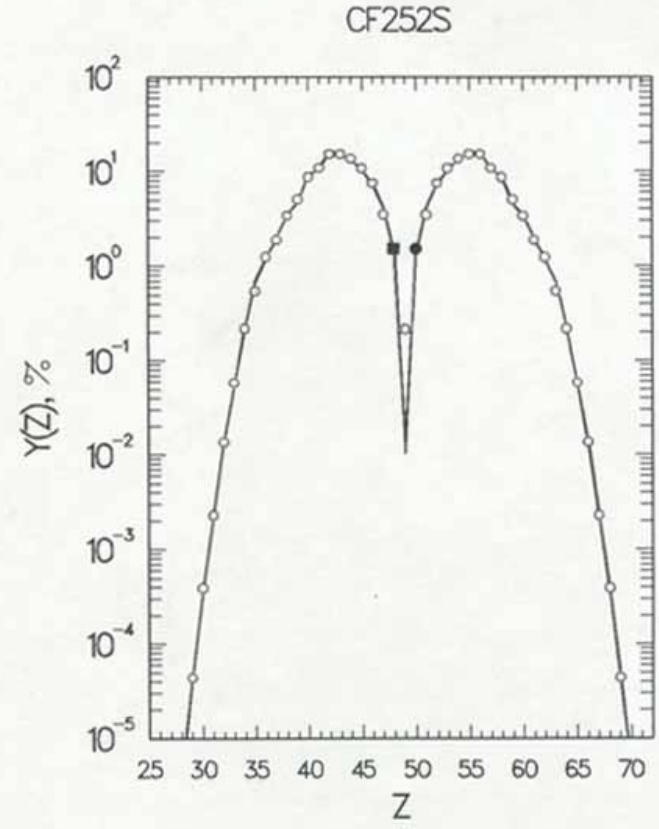


Fig. 2. Element yields $Y(Z)$ vs. mass number $Z$. $\circ \bullet \mathbf{n}$ : calculated from the recommended independent yields based on the $Z_{p}$ model. $\bullet: Z=50 ; \mathbf{m}: Z=Z_{F}-50$. - : calculated from the $A_{p}$ model [12].

\section{Calculation of the amount of source material necessary}

The decision about the amount of californium used for the source should be influenced by two important factors. On the one hand the amount should be sufficient to obtain a reasonable amount of the radionuclides of interest. On the other hand the amount should be not larger then necessary to decrease the amount radiation that needs to be shielded and to keep the costs of the generator as low as possible. The following calculations are based on the assumption that $4 \mu \mathrm{g}$ of ${ }^{252} \mathrm{Cf}$ are used as source material.

An amount of $4 \mu \mathrm{g}$ contains about $9.56 \times 10^{15}$ atoms of ${ }^{252} \mathrm{Cf}$. Based on a half-life of 2.6 years this means that the source undergoes a total number of about $80,000,000$ decays per second. Since only $3 \%$ of the decays are fission events, the nuclides contained in the source would fission about 2,400,000 times per second. This means about 19296 atoms of ${ }^{112} \mathrm{Pd}$ are produced per second.

A smaller amount of ${ }^{252} \mathrm{Cf}$ would be easier to handle while preparing the source and would lower the overall radiation. But using less material for the source would result in a substantially lower amount of fission products. An amount of $4 \mu \mathrm{g}$ allows for the sufficient production of nuclides with short and medium half-lives that can be used for various types of experiments.

\section{Usefulness of the fission products obtained}

As already mentioned before the spontaneous fission of ${ }^{252} \mathrm{Cf}$ yields mainly shortlived isotopes with mass numbers between 90 to 120 and 130 to 160 . Fig. 3 shows cut outs of the chart of nuclides in the appropriate mass regions. The nuclides marked red have been detected using an existing fission product generator [13].

The main fission products include isotopes of $\mathrm{Zr}, \mathrm{Nb}, \mathrm{Mo}, \mathrm{Tc}, \mathrm{Rh}$ and $\mathrm{Rh}$ with halflives of up to a few seconds. These elements are homologues of the transactinide elements Rf, $\mathrm{Db}, \mathrm{Sg}, \mathrm{Bh}, \mathrm{Hs}$ and Mt, elements 104 to 109. Homologues play an important role in the development of model systems to study heavy elements. The study of the homologues is essential to compare the chemical behavior of the transactinides to other elements. 

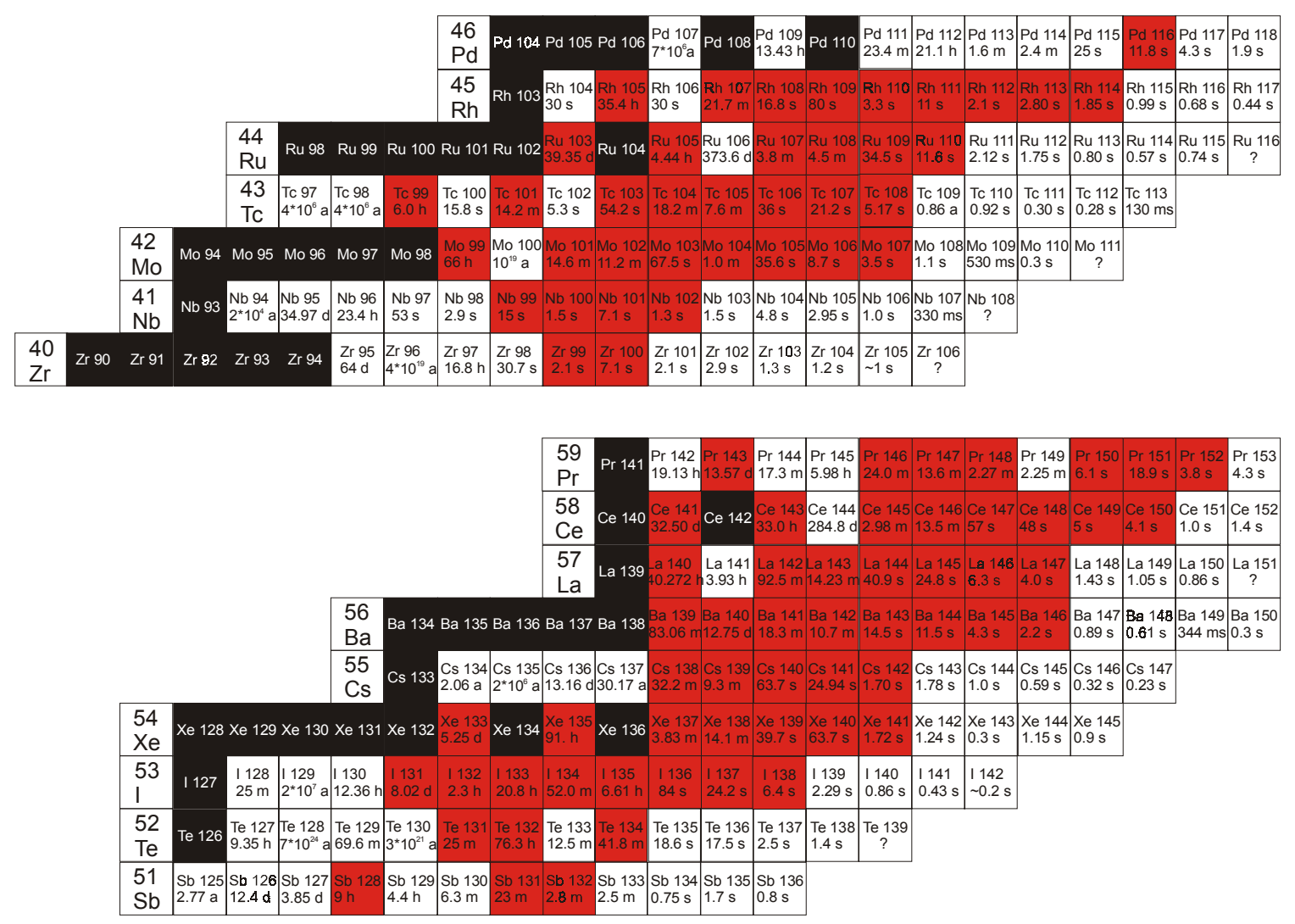

Fig. 3. Cut out of the chart of nuclides that show the light and heavy mass peaks of the ${ }^{252} \mathrm{Cf}$ fission products. $\mathrm{m}$ : stable nuclides; $\mathbf{a}$ : nuclides detected with other fission product generators using a ${ }^{252} \mathrm{Cf}$ source. [13]

The half-lives in the range of seconds make these nuclides ideally suited to be used for gas phase chemistry experiments or for experiments using fast, automated setups for aqueous chemistry like the SISAK system.

The heavy mass peak of the fission product distribution includes elements like $\mathrm{Sb}, \mathrm{Te}$ and I. These elements could be considered as the homologues of even heavier transactinide elements like element 115,116 or 117 . Although at this point the study of these transactinide elements is not planned yet, eventual studies will start out by studying the proposed homologues. The fission products of the heavy branch of the mass yield curve could play an important role in the development of new fast chemistries suitable to study these heavy transactinide elements in future experiments.

The planned radionuclide generator should also be suitable to provide radiotracers. These tracers can be used in a multitude of different experiments. Longer-lived radionuclides can be obtained from the fission source by collecting the primary fission products and allowing for them to undergo multiple $\beta^{-}$decays towards more stable nuclei.

The cumulative fission yield for a specific isotope is given by the sum of the independent fission yields of all isotopes that decay to this specific isotope. So in general the cumulative yield is a lot higher than the independent yield. Many radioisotopes of interest, like ${ }^{95} \mathrm{Zr}$, have only a very small independent fission yield, but their cumulative 
yield is significant. Table I. shows the independent and cumulative yields for several isotopes that might be useful as radiotracers together with their respective half-lives. The time that is necessary to collect a sufficient amount of each radiotracer depends on the time that is necessary for the decay chain to reach equilibrium. After the equilibrium is reached, 31200 atoms of ${ }^{95} \mathrm{Zr}$ per second are produced instead of 312 .

Table I. Half-lives, independent yield and cumulative yields for selected ${ }^{252} \mathrm{Cf}$ fission products that can be used as radioactive tracers.

\begin{tabular}{|c|c|c|c|} 
Nuclide & Half-life & Independent yield & Cumulative yield \\
\hline${ }^{95} \mathrm{Zr}$ & $64 \mathrm{~d}$ & $0.013 \%$ & $1.300 \%$ \\
\hline${ }^{96} \mathrm{Nb}$ & $23.4 \mathrm{~h}$ & $0.0005 \%$ & $1.534 \%$ \\
\hline${ }^{99} \mathrm{Mo}$ & $66 \mathrm{~h}$ & $0.002 \%$ & $2.682 \%$ \\
\hline${ }^{99} \mathrm{Tc}$ & $6.0 \mathrm{~h}$ & $0.000001 \%$ & $2.682 \%$ \\
\hline${ }^{103} \mathrm{Ru}$ & $39.35 \mathrm{~h}$ & $0.0003 \%$ & $5.465 \%$ \\
\hline${ }^{105} \mathrm{Rh}$ & $35.4 \mathrm{~h}$ & $0.00006 \%$ & $6.164 \%$ \\
\hline${ }^{112} \mathrm{Pd}$ & $21.2 \mathrm{~h}$ & $0.804 \%$ & $4.284 \%$ \\
\hline${ }^{143} \mathrm{Pr}$ & $13.57 \mathrm{~d}$ & $0.00002 \%$ & $6.434 \%$ \\
\hline${ }^{144} \mathrm{Ce}$ & $284.8 \mathrm{~d}$ & $0.195 \%$ & $5.921 \%$ \\
\hline${ }^{140} \mathrm{La}$ & $40.272 \mathrm{~h}$ & $0.006 \%$ & $6.336 \%$ \\
\hline${ }^{140} \mathrm{Ba}$ & $12.75 \mathrm{~d}$ & $0.509 \%$ & $6.330 \%$ \\
\hline${ }^{137} \mathrm{Cs}$ & $30.17 \mathrm{a}$ & $0.212 \%$ & $4.931 \%$ \\
\hline${ }^{131} \mathrm{I}$ & $8.02 \mathrm{~d}$ & $0.003 \%$ & $1.388 \%$ \\
\hline${ }^{132} \mathrm{Te}$ & $76.3 \mathrm{~h}$ & $0.728 \%$ & $1.947 \%$ \\
\hline${ }^{126} \mathrm{Sb}$ & $12.4 \mathrm{~d}$ & $0.0002 \%$ & $0.027 \%$
\end{tabular}

The production of short-lived and long-lived radiotracers from a ${ }^{252} \mathrm{Cf}$ fission fragment source presents the opportunity for performing heavy element homologue chemistry experiments in an off-line environment that is similar to the on-line chemistry environment. These efforts will save research time and money that would be otherwise spent on extremely valuable Cyclotron irradiation time, where mistakes and errors can prove costly. By developing systems in the off-line environment, when the time comes for an on-line experiment, the Cyclotron irradiation time can be used to its maximum extent. The construction of a ${ }^{252} \mathrm{Cf}$ fission fragment source is the realization of this offline environment.

\section{Design}

\section{A. History}

Dieter Jost and colleagues at PSI (Paul Scherrer Institut) in Switzerland built a device for the capture of the fission fragments from a ${ }^{252} \mathrm{Cf}$ source [1]. Their device, named "Miss Piggy" was designed to collect the fission fragments from a ${ }^{252} \mathrm{Cf}$ source and use them in gas chemistry apparatus, studying the chemical behavior of the homologues of the transactinide elements (see Fig. 1). 


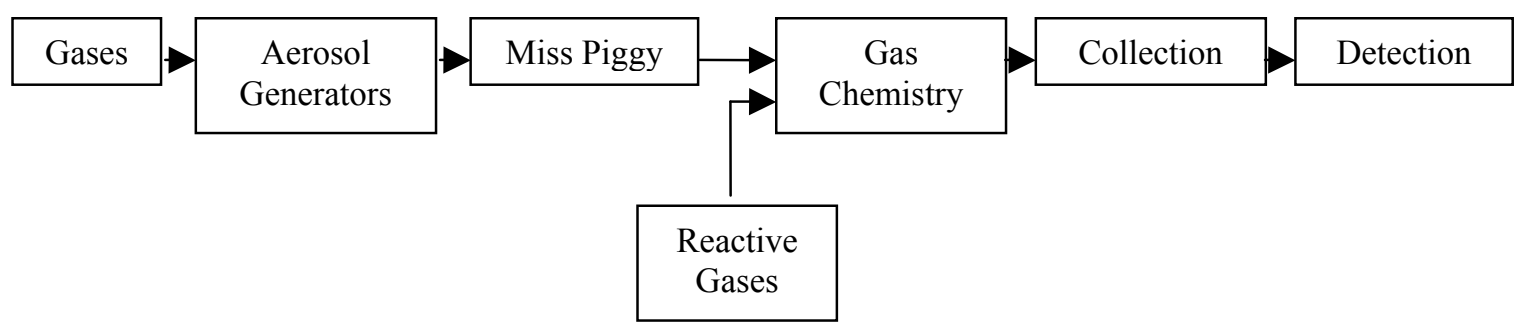

Fig. 1. Flowchart of the Miss Piggy apparatus.

Their device was adaptable to multiple transport gases and reaction chamber pressures. The ${ }^{252} \mathrm{Cf}$ was electroplated on a platinum foil and covered with a thin aluminum foil. For safety reasons, a second foil was placed between the source and the reaction chamber, separating the ${ }^{252} \mathrm{Cf}$ source from the reaction chamber. The ${ }^{252} \mathrm{Cf}$ source could be placed in two different positions, a park position and a running position. In the park position, the fission fragments recoiled into the shielding. In the running position, the fission fragments recoiled from the source into the reaction chamber. The volume of the chamber was adjusted for the gas pressure and type of transport gas used. These recoils were then transported using a gas-jet system to the chemical study apparatus [2]. A PC was used to control the running parameters of "Miss Piggy" and to display the system status at all times. The reaction chamber was contained in a one meter steel ball, filled with polyethylene and borated paraffin to provide adequate neutron shielding. Design drawings can be found in Appendix A.

At the time of the Scientific Report, a second-generation device was being built. Called "Kermit" this device was to be similar to "Miss Piggy", but with a couple of alterations. The reaction chamber was going to be built to resist the reaction gases used in the chemistry apparatus and was to be modified to be able to mount the gas chemistry apparatus directly to the reaction chamber, eliminating the need for a gas transport system to the chemistry apparatus. These modifications also enlarge the number of nuclides that could be studied by shortening the time between fission fragment emission and gas chemistry, allowing the study of shorter lived isotopes.

\section{B. Goals}

The BORG design goals are to make fundamental improvements to existing designs, to make the device adaptable to multiple environments, to make the device modular for easy interchange of components, and to make the device safe and easy to use.

The major areas of improvement over previous designs are the incorporation of a third position for the ${ }^{252} \mathrm{Cf}$ source, a modular reaction chamber and components, and a possible third bore for neutron activation. The original designs only had two positions, a storage position and a running position. The BORG will incorporate a third position, or the long half-life collection position. In this position, fission fragments will recoil on to a catcher foil that can be removed from the apparatus. The generated activity can be separated from the catcher foil and used in various chemistry experiments. The reaction 
chamber will be built with the idea that it can be removed and replaced with other devices for different chemistry experiments (i.e. Mass Spectrometry) and for ease in cleaning. The capillaries used inside the BORG will be easily replaced to make cleaning simple. Finally, not only are the homologues of the transactinides important for heavy element chemistry. Neutron activation of stable isotopes allows the production of long-lived tracers that can be used in various radiochemical experiments. These experiments are used in the development of new techniques for the study of the heaviest elements. For example, it is important to use lanthanide tracers to study the chemical behavior of the heavier actinide elements. Neutron activation of specific lanthanides will allow the study of the chemistry of the heavier actinides.

\section{Overview}

Our design for the BORG could be considered a close relative of "Miss Piggy" and "Kermit." However, we propose to make changes to those simple designs that will make the BORG a more prominent tool in the study of the homologues of the transactinide elements.

The most important design goal for the BORG is the transportation of the ${ }^{252} \mathrm{Cf}$ fission fragments from the source to the chemistry apparatus in a safe and easy to use manner. Fig. 2 illustrates the design idea for the BORG that will best accomplish this goal.
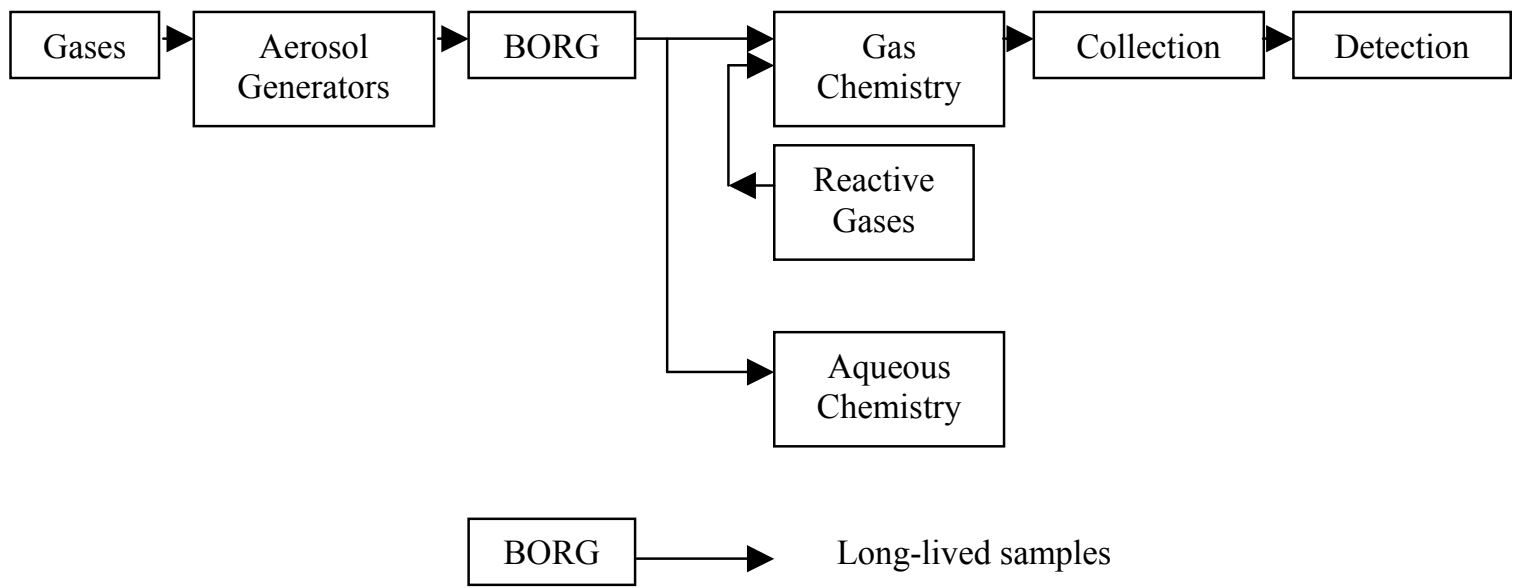

Fig. 2. Flowchart for the BORG apparatus.

\section{Berkeley Off-line Radioisotope Generator}

The centerpiece of the fission fragment collection apparatus is the ${ }^{252} \mathrm{Cf}$ source and source housing. The housing for the ${ }^{252} \mathrm{Cf}$ will be a cube of polyethylene called the Berkeley Off-line Radioisotope Generator, or BORG. Gas and aerosols will be transported to the reaction chamber of the BORG. A PC program (LabView) will control gas pressure, flow rate and composition. An appropriate aerosol will be used for the transport of the fission fragments from the reaction chamber to the chemistry apparatus. The BORG will be designed to accommodate various aerosols such as potassium 
chloride, sodium chloride, and carbon. The BORG will consist of 48 sheets of polypropylene, each 48 inches by 48 inches by 1 inch, sandwiched between a steel base and a steel support frame on top. The steel supports will be secured together through the use of four large screws in the four corners of the cube. Each sheet of polypropylene will be machined to accommodate the reaction chamber, storage position, and long-lived isotope position. The ${ }^{252} \mathrm{Cf}$ will be electrodeposited onto a $\mathrm{Pt}$ foil and covered with an $\mathrm{Al}$ foil to prevent source leakage. A second foil will be used to separate the running position from the reaction chamber. Finally, a third foil will be located at the bottom of the reaction chamber. These foils will be used together to reduce contamination. Additional work will be done on the polypropylene to make room for the capillaries to connect the reaction chamber to the outside of the BORG, for the long half-life position bore, and for the neutron activation bore. The reaction chamber will be built in a similar fashion to the reaction chamber built by the group at PSI (see Appendix A). All of the new modifications will also be built in a similar style. A PC program will control the safety interlocks, ${ }^{252} \mathrm{Cf}$ source position, reaction chamber pressure, and flow rate through the reaction chamber.

The fission fragments laden aerosols then make it through the capillary to the outside of the BORG where they can be directed to one of four capillaries. These four capillaries would head towards the gas chemistry apparatus, the aqueous chemistry apparatus, the yield check system, and a waste system. The gas chemistry and aqueous chemistry systems will be located in hoods in the same room as the BORG. The yield check system will be a direct line to the collection and detection site. The handling of waste products from the BORG will be done in accordance to all existing EH\&S safety and waste management procedures.

\section{Collection and Detection}

The gas chemistry and aqueous chemistry systems used with the BORG will be similar to those already used [2-11]. The collection and detection system, however, will be brand new. The collection/detection system, called the MiniVW (mini vertical wheel), will be used for the measurement of fission fragment activity from the gas chemistry. It will consist of a mini rotating wheel and two low-energy gamma ray detectors. Fig. 3 shows the concept for the MiniVW. 


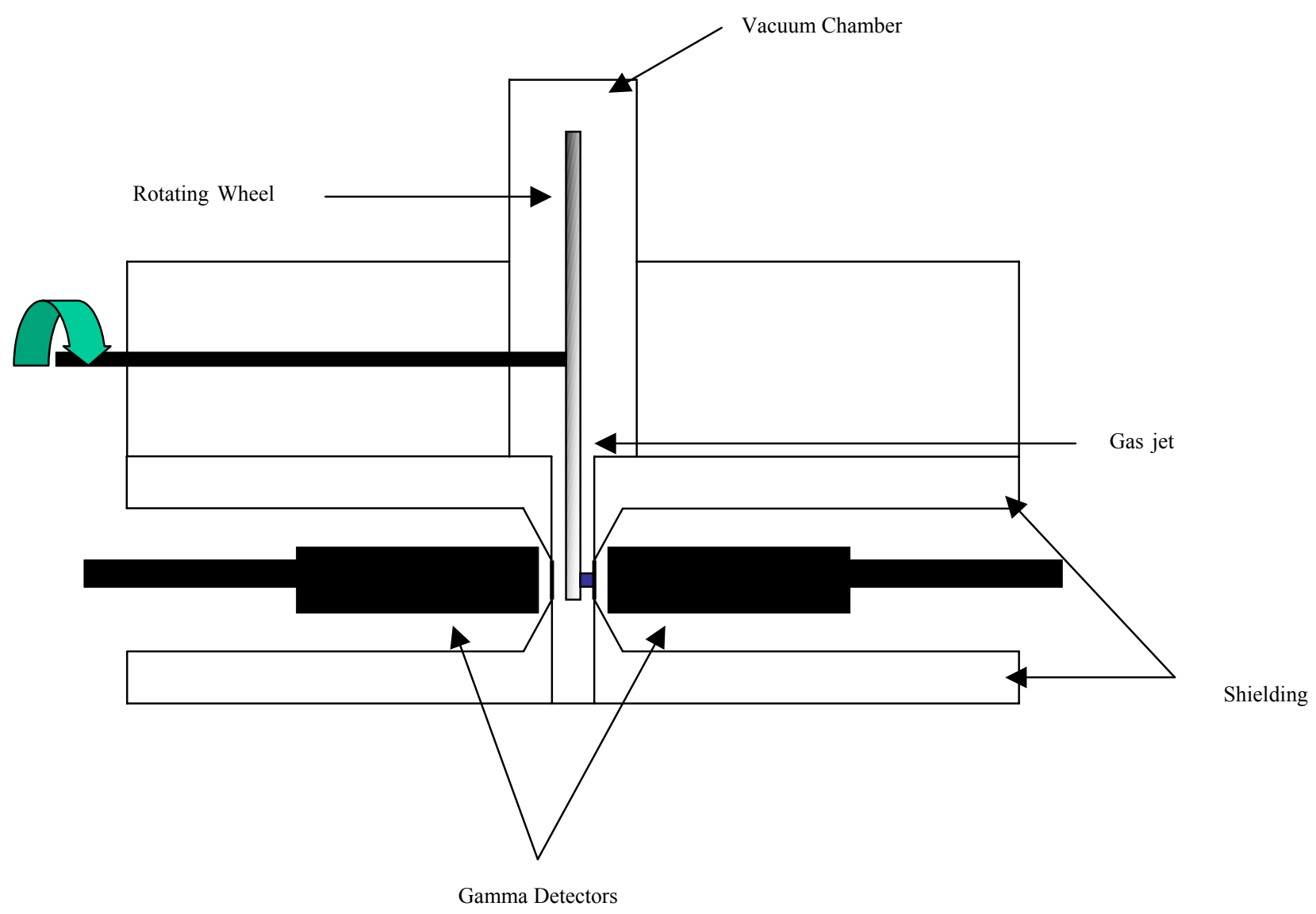

Fig. 3. The MiniVW design.

The chemistry products will be transported through a capillary to the MiniVW and deposited on a vertically mounted wheel. Various wheels will be used to collect the activity. The aerosols used to transport the activity will determine what type of material the wheel will be made of. For example, a polypropylene wheel would be used to collect $\mathrm{KCl}$ aerosols. A PC based stepping program will be used to control the motion of the wheel to move the deposited activity between the gamma detectors. The vacuum chamber will be kept small to decrease the amount of time necessary to pump the chamber down. Thin Mylar foils will be used between the vacuum chamber and the gamma detectors.

\section{Safety}

All of the major systems within the BORG apparatus will be controlled by a PC system. Gas composition and flow rates as well as BORG interlock systems will be controlled by one PC system, while the collection/detection system will be controlled by 
a second system. Adequate safety monitoring will be performed to detect any unintentional release of fission fragment activity. Shielding calculations were performed and are summarized in Appendix B. Additional neutron and gamma shielding will be used beyond what is suggested by the shielding calculations. Communication between our group and the proper EH\&S authorities will be maintained at each step in the construction and commissioning.

\section{Summary}

The proposed radionuclide generator BORG is a valuable source to obtain carrier free tracers with short and medium half-lives. These radiotracers can be used to perform various types of liquid and gas phase chemistry. They are an essential part in performing homologue experiments to study the presently transactinide elements.

Short-lived radionuclides can be transported directly from the source to experimental setups using a gas jet system. Medium-lived radionuclides can be collected from the source on a catcher foil, removed from the generator and processes afterwards. In addition other types of nuclides can be obtained by neutron activation. The fission products available from the ${ }^{252} \mathrm{Cf}$ source include some of the most important homologue elements for transactinide studies. The radionuclides can be produced in sufficient quantity using a $4 \mu \mathrm{g}{ }^{252} \mathrm{Cf}$ source.

The modular design of the device allows for the adaptability and flexibility of the system and will also leave room for future improvements.

The proposed design of the system and the shielding will allow for an easy and safe use of the generator in a laboratory and will ensure that no unnecessary exposure to radiation can occur. This is ensured by a combination of administrative and engineering procedures that include the use of interlocks and the permanent monitoring of the system using computers.

The BORG system should enable the Heavy Element Group to perform invaluable experiments to study even heavier transactinide elements in addition to allowing broadening the scope of present experiments. The BORG system should enable the group to do work on the chemical homologues of the transactinide elements without the need to limit the work to available Cyclotron beam time. 


\section{Appendix A}

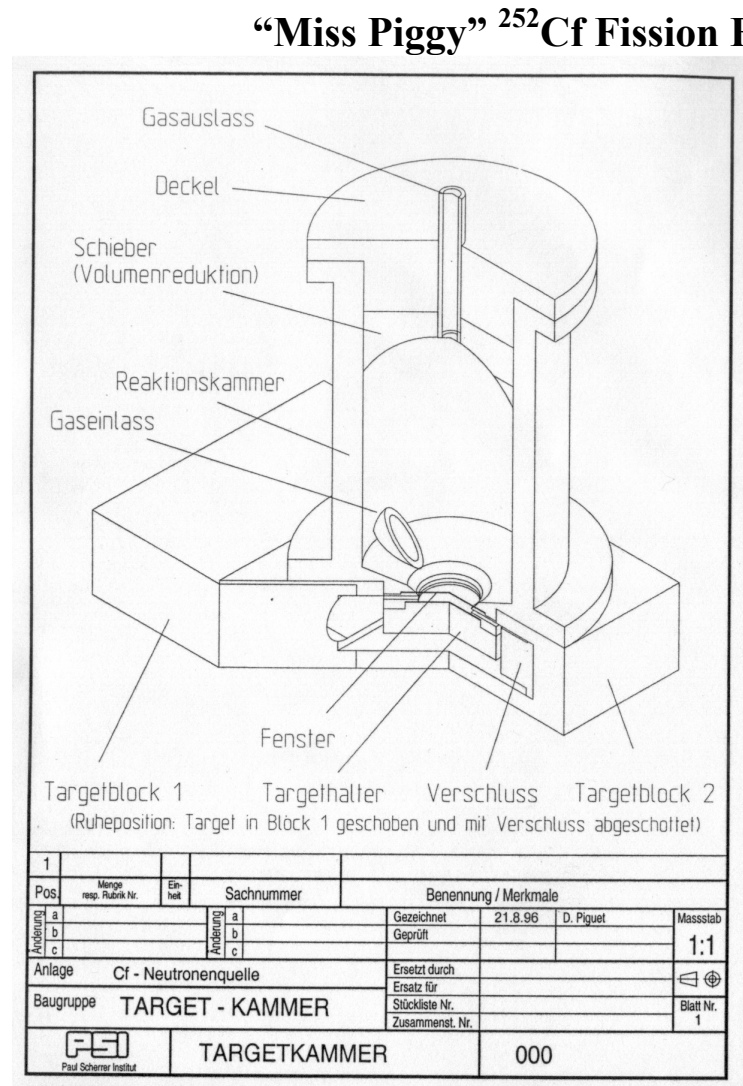

Three-dimensional drawing of the reaction chamber.

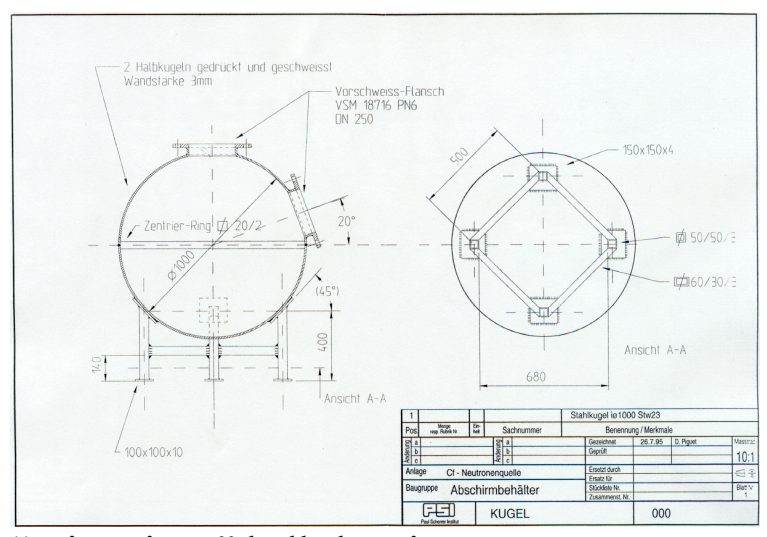

"Miss Piggy" ball showing support structure.

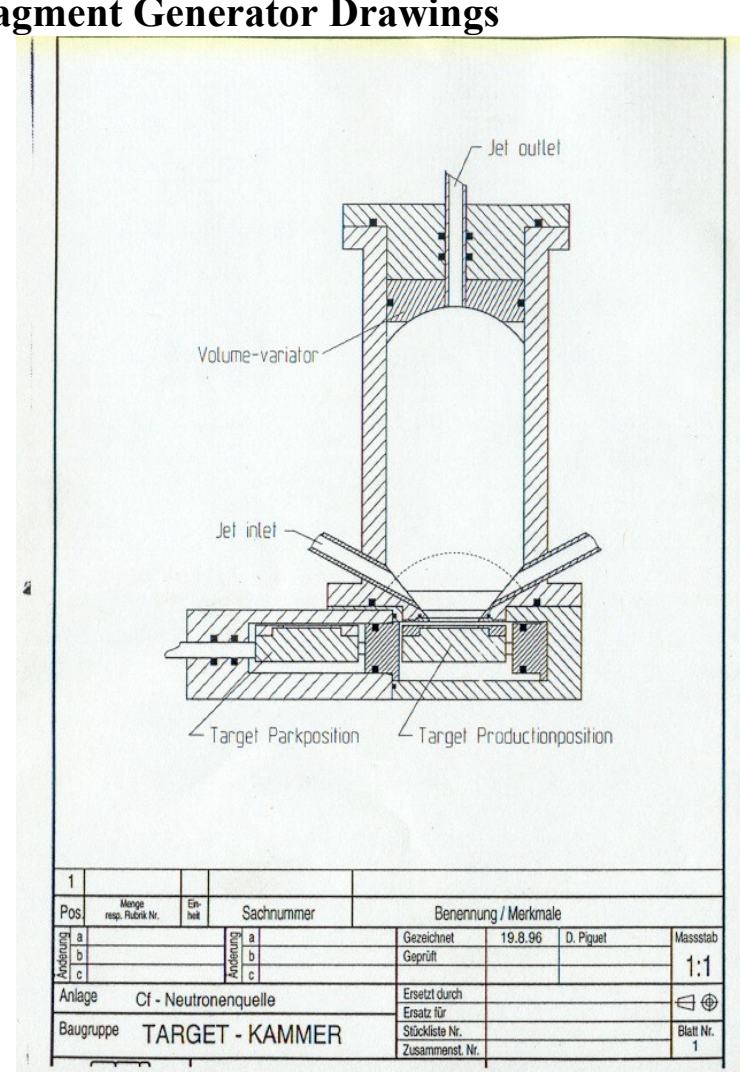

Side view of reaction chamber showing only two source positions.

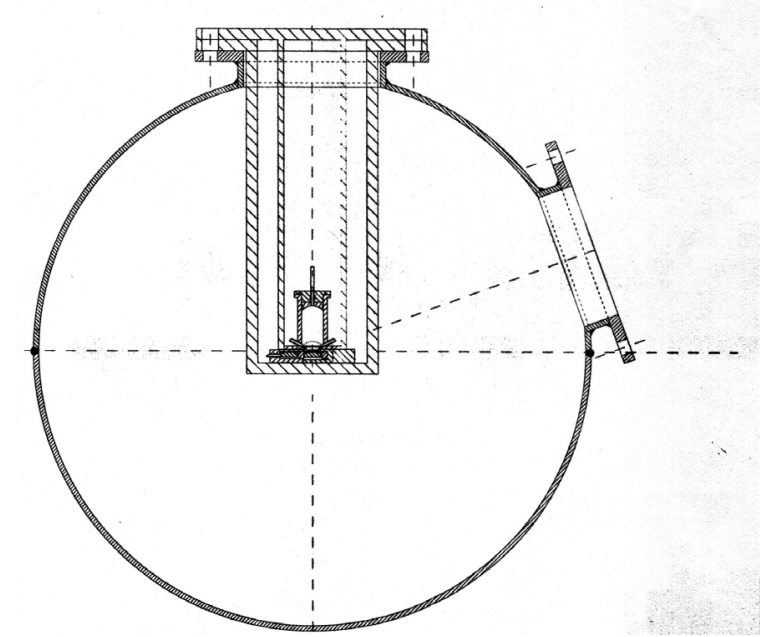

"Miss Piggy" showing relative size of sphere to the reaction chamber.

All drawings courtesy of Dr. Andreas Türler, Paul Scherrer Institut, Switzerland. 


\section{Appendix B}

\section{Shielding Calculations}

At the outset of the project, communication between Rick Donahue of LBNL EH\&S and our group was begun to determine the appropriate amount of shielding required for adequate protection from neutrons and gamma activity from a 4 microgram ${ }^{252} \mathrm{Cf}$ source.

Rick performed a quick analysis of a ${ }^{252} \mathrm{Cf}$ source inside a spherical polyethylene moderator. He assumed 4 micrograms of ${ }^{252} \mathrm{Cf}$ or $9.2 \times 10^{6}$ neutrons/second. He sampled the energy from a Watt fission spectrum with constants $a=1.025(\mathrm{MeV})$ and $b=2.926$ $(1 / \mathrm{MeV})$. He used compositions of polyethylene and borated polyethylene from a supplier. The dose rates shown below are calculated on the outside of the surface of the shield.

\begin{tabular}{|c|c|c|c|c|c|c|c|}
\hline & & & Do & ates & & & \\
\hline & $30 \mathrm{~cm} \mathrm{P}$ & ethylene & & & $50 \mathrm{~cm} \mathrm{P}$ & ethylene & \\
\hline$\underline{\text { Case }}$ & $\underline{\text { Neutron }}$ & $\underline{\text { Gamma }}$ & Total & $\underline{\text { Case }}$ & $\underline{\text { Neutron }}$ & $\underline{\text { Gamma }}$ & $\underline{\text { Total }}$ \\
\hline No B & 0.99 & 0.76 & 1.75 & No B & 0.01 & 0.17 & 0.18 \\
\hline $5 \% \mathrm{~B}$ & 1.08 & 0.14 & 1.22 & $5 \% \mathrm{~B}$ & 0.04 & 0.02 & 0.06 \\
\hline
\end{tabular}

All values are in mrem/hour.

Neutrons are effectively shielded after about $30 \mathrm{~cm}$ of polyethylene. Thermal neutron capture by hydrogen produces a $2.2 \mathrm{MeV}$ gamma ray that then dominates. These gamma rays could be shielded by approximately 2 inches of lead, or the substitution of polyethylene with $5 \%$ borated polyethylene. $5 \%$ borated polyethylene is approximately twice the cost of normal polyethylene.

Rick suggested $50 \mathrm{~cm}$ of $5 \%$ borated polyethylene as an appropriate shield for 4 micrograms of ${ }^{252} \mathrm{Cf}$. Linnea Wahl, a health physicist with LBNL EH\&S, examined these does rates and informed the group that not only would $50 \mathrm{~cm}$ of $5 \%$ borated polyethylene be perfectly fine, but also that $50 \mathrm{~cm}$ of regular polyethylene be fine.

Additional lead and polyethylene will be used in the construction of the BORG beyond what is suggested in these dose rate calculations, using additional polyethylene as well as lead shielding already present in the lab. 


\section{References}

[1] D.T. Jost, et al, PSI Scientific Report 1998, Volume 1, 120 (1999)

[2] A. Türler, Radiochim. Acta 72, 7 (1996)

[3] E. Sylwester et al, Radiochim. Acta 88, 837 (2000)

[4] R. Eichler et al, Nature 407, 63 (2000)

[5] U. Kirbach, submitted to NIM A (2001)

[6] R. Eichler et al, Radiochim. Acta 87, 151 (1999)

[7] R. Eichler et al, Radiochim. Acta 88, 87 (2000)

[8] C.D. Kacher et al, Radiochim. Acta 75, 127 (1996)

[9] R. Günther et al, Radiochim. Acta 80, 121 (1998)

[10] J.P. Omtvedt et al, J. Alloys Comp. 271-273, 303 (1998)

[11] B. Wierczinski et al, J. Radio. Nucl. Chem. 247, 57 (2001)

[12] A.W. Wahl, Atom. Data Nucl. Data Tables 39, (1988)

[13] Ch. Düllmann et al, PSI Scientific Report 1998, Volume 1, 121 (1999)

[14] R. J. Silva, et al, Nucl. Inst. Meth. 147, 371 (1977) 